\title{
New local optics measurements and correction techniques for the LHC and its luminosity upgrade
}

\author{
J. Coello de Portugal@, ${ }^{*}$ R. Tomás, and M. Hofer \\ CERN, 1211 Geneva, Switzerland
}

(Received 2 May 2019; revised manuscript received 9 March 2020; accepted 23 March 2020; published 6 April 2020)

\begin{abstract}
As the beams at the interaction points (IPs) of circular colliders are pushed toward smaller sizes, the correction of the magnetic field errors in high- $\beta$ regions become increasingly important, but also challenging. This paper presents an algorithm developed to compute automatically local corrections. This algorithm has been successfully used in the LHC and in simulations of the HL-LHC to establish tolerances for the magnetic errors. The limitations of the current $\beta^{*}$ measurement technique (K-modulation) are studied, together with alternative techniques for $\beta^{*}$ control: computing the minimum $\beta$ near the IP using the betatron phase measured with new instrumentation and locating the beam waist via luminosity scans. This push toward smaller beam sizes also requires large $\beta$-functions in the arcs that enhance local errors currently negligible. Experimental results of a way of correcting this new type of local errors using orbit bumps in sextupoles is also presented. These studies forecast a drastic change in the LHC commissioning strategy to be applied in the HL-LHC for needing luminosity measurements in intermediate stages.
\end{abstract}

DOI: 10.1103/PhysRevAccelBeams.23.041001

\section{INTRODUCTION}

During the LHC Run 2, the optics corrections have brought the global r.m.s. $\beta$-beating in the machine to the $2 \%$ level and the r.m.s. $\beta^{*}$-beating down to the $1 \%$ level [1-3]. However, the continuous effort to reduce the beam sizes at the interaction points is starting to bring up the limitations of current optics measurements and correction methods. For HL-LHC this is viewed as one of the main challenges from the optics correction point of view [4]. A failure to control local optics in strongly squeezed configurations can lead to reduced luminosity and to a luminosity imbalance between ATLAS [5] and CMS [6] (and ALICE [7] in case of ion physics). Accurate optics control is also fundamental to ensure the machine safety.

$\mathrm{K}$-modulation has been the tool used to measure $\beta^{*}$ and waist shifts during LHC Run 2, which has been essential to achieve an unprecedented performance of the machine during the run. Recent measurements during experimental studies at low- $\beta^{*}$ and flat optics (different horizontal and vertical $\beta^{*}$ ) have revealed significant limitations in $\beta^{*}$ measurements for these optics regimes. Also, theoretical studies for HL-LHC [8,9] show that K-modulation will not reach the precision requirements to ensure the expected

\footnotetext{
*Also at Universitat Politècnica de Catalunya

Published by the American Physical Society under the terms of the Creative Commons Attribution 4.0 International license. Further distribution of this work must maintain attribution to the author(s) and the published article's title, journal citation, and DOI.
}

performance of the machine. Therefore, for the future of the LHC it will be essential to count on alternative methods for $\beta^{*}$ control to complement or replace K-modulation.

The Achromatic Telescopic Squeeze (ATS) scheme [10] has become operational in the LHC and it is the baseline for HL-LHC. This optics scheme uses the arcs around the low$\beta^{*}$ insertions, namely arcs $81 / 12$ and $\operatorname{arcs} 45 / 56$, to aid the matching section quadrupoles to match the optics in the interaction regions and correct the chromatic effects by allowing an increase of the $\beta$-function in these arcs, peaking at lattice sextupoles. As a downside, this increase of the $\beta$-function in the arcs also enhances the effects from magnetic or alignment errors and affects the optics quality. The lack of individually powered quadrupoles in the arcs makes these errors very challenging to correct.

This paper presents experimental progress on techniques to improve the performance of optics local corrections in Run 3 and in HL-LHC. A way of correcting the enhanced local errors in the arcs caused by strong ATS squeeze is to use orbit bumps in sextupoles to produce a quadrupolar fields via feed-down. First experimental results of this technique, performed during machine development studies in the LHC, are presented here.

In Sec. II the need and implementation of an automatic local-optics correction tool are discussed together with performance results from measurements and simulations. In Sec. III the issues found experimentally in K-modulation measurements together with possible mitigation techniques are presented. In Sec. IV an alternative to K-modulation to measure the minimum $\beta$-function in the interaction regions using phase-advance is presented. In Section V a 
complementary method to find the location of the beam waist by displacing it and measuring luminosity is also presented. In Sec. VI the large $\beta$-beating produced by local errors in the high-beta ATS arcs observed in the LHC and expected for HL-LHC are shown and the implemented and foreseen solutions are discussed.

\section{LOCAL OPTICS CORRECTIONS}

Traditionally, the betatron phase-advance has been the most robust optics observable as it is independent of beam position monitor (BPM) calibration and tilt errors and it does not rely on a model of the machine. Local phase errors in the interaction regions (IR) have been successfully corrected using the segment-by-segment technique $[11,12]$. The measured phase-advance in chosen segments of the accelerator can be compared to a model of the segment using MAD-X [13], whose initial conditions are set from measurements at the initial point of the segment. In this way, only quadrupolar errors within the segment affect the phase beating between measurement and propagated model, as shown in Fig. 1. The model of the segment is matched to the local phase deviations by trimming the quadrupoles in the region. By swapping the sign of these trims an effective correction is obtained.

This matching, was traditionally performed manually by trial-and-error which required a large human effort. An automatic tool has been developed that solves these challenges: (i) As the two beams traverse a common set of quadrupoles in the triplets region, a common correction must be found for both beams. (ii) The measurement of the $\beta$ function from K-modulation at the IP has been introduced as a constraint, to avoid significant waist shifts and must also be matched simultaneously. (iii) For local coupling correction not only both beams must be matched simultaneously, but also the real and imaginary parts of the $f_{1001}$ resonance driving term. (iv) In HL-LHC $\beta^{*}$ levelling will be used [14]. This will require us to commission up to 50 different optics [15] which would be a extremely hard task to perform manually. The automatic tool is much faster in performing

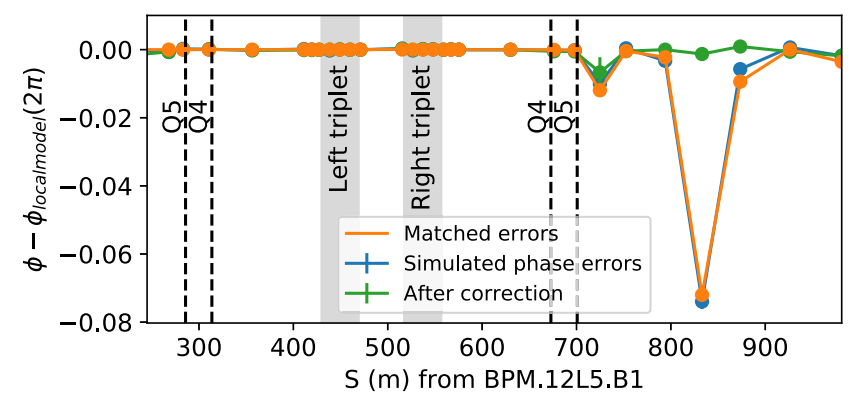

FIG. 1. Example of a simulation of the HL-LHC with quadrupolar errors in the triplet and Q4-5. The blue line shows the deviation of the phase-advance from the design model of the segment. The orange line is the suggested effective correction and the green the effect of the correction. the corrections for each optics. (v) It improves the accuracy and reproducibility of the corrections.

\section{A. Automatic local matching}

The local-model matching can be performed as an optimization problem in MAD-X by setting all measurement points for both beams in the segment as constraints and all available quadrupole strengths as variables. These algorithms have been extensively tested in HL-LHC simulations and put in practice in LHC optics commissioning and dedicated experiments.

In Fig. 1 an example of the behavior of the automatic matching tool on the HL-LHC lattice is shown. The triplet quadrupoles and the two matching quadrupoles (Q4-5) in each side (see layout on top of Fig. 2) of the interaction point (IP) are given random $10^{-3}$ relative gradient error and the triplet circuits are used as variables to compensate the errors. The target solution is constrained by the measured deviation of phase-advance and $\beta$-functions from K-modulation for both beams. The blue line shows the simulated deviation of the phase-advance from the local model of the segment and in orange the suggested match performed by MAD-X optimization. The green line shows the local errors remaining after applying the suggested matched values with its sign reversed.

The same technique can be used to correct local coupling by targeting the $f_{1001}$ resonance driving term [12] as shown in Fig. 3. These local coupling errors are caused by random tilts of the triplet quadrupoles and Q4-5, producing coupling via feed-down. The coupling is corrected using the 2 skew quadrupoles in the IR (Fig. 2 top).

The importance of a good local coupling correction was brought to view in the ions run of 2018, where a human error caused the swap of the left and right corrector strengths given by this technique and produced a $50 \%$ loss of luminosity in ALICE [16].

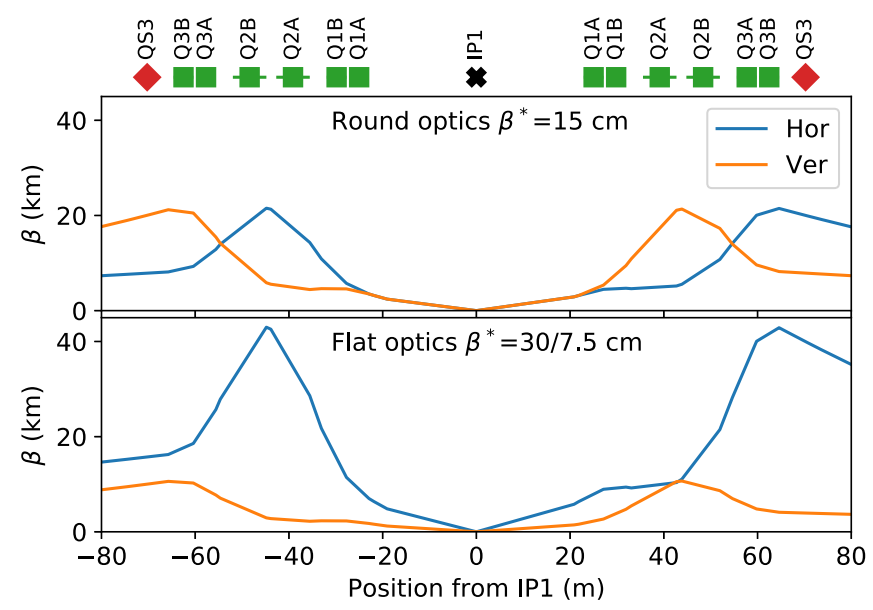

FIG. 2. Schematic of the quadrupoles layout and $\beta$-function in the triplet region of HL-LHC around IP1 for round and flat optics. 


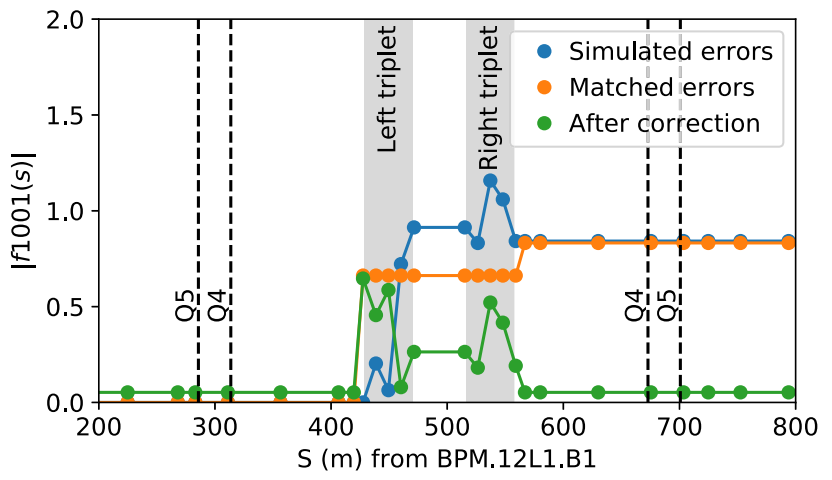

FIG. 3. Example of a simulation of HL-LHC with roll errors in the triplet and Q4-5 quadrupoles. The effect of these errors in the $\left|f_{1001}\right|$ is shown in blue. The suggested effective correction is shown in orange, but it should be noted that the matching routine matches the real and imaginary parts of the $f_{1001}$ resonance driving term. The effect of the correction is shown in green.

\section{B. $\beta^{*}$ corrections performance in the HL-LHC}

To assess the performance of this technique in the HLLHC, we performed Monte-Carlo simulations of the expected field and alignments errors in the interaction region quadrupoles, and applied the automatic correction describe above to each of the simulation seeds.

Specifically, each quadrupole of the triplet and Q4-5 is given a random $10^{-3}$ relative quadrupolar error from a Gaussian distribution and a $\pm 2 \mathrm{~mm}$ longitudinal misalignment. Sorting is applied to the two pieces of the Q2 quadrupoles, to avoid big relative field imbalances between these two pieces.

The resulting phase-advance deviations in the IP1 and IP5 segments are also disturbed with Gaussian noise to simulate the measurement noise. This noise is assumed to be $6 \mathrm{mrad}$ r.m.s. at a BPM with $\beta=171 \mathrm{~m}$ and scaled to the rest with the $\sqrt{\beta}$ at their location.

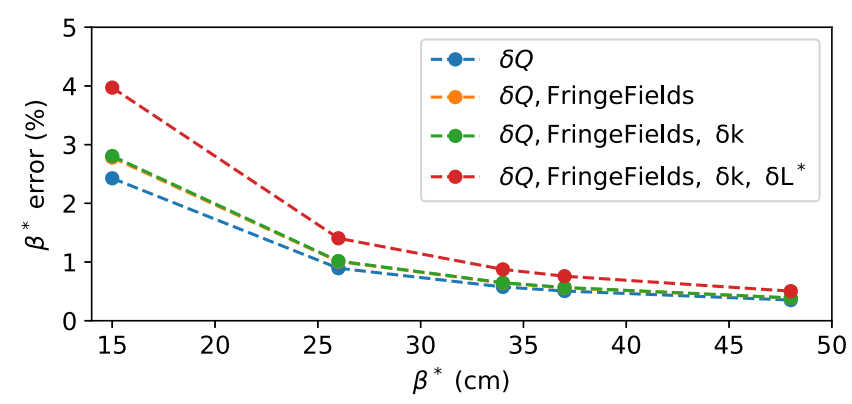

FIG. 4. Simulation of the evolution of the $\beta^{*}$ error from Kmodulation with the design $\beta^{*}$. The effect of a tune uncertainty of $2.5 \times 10^{-5}$ is shown in the blue line. The orange line (partially covered by the green line) also considers the modulating quadrupole fringe fields. In the green and red lines a $10^{-3}$ relative uncertainty in the field of the modulating quadrupole and a $2 \mathrm{~mm}$ uncertainty in the longitudinal position of the quadrupole have been respectively added.

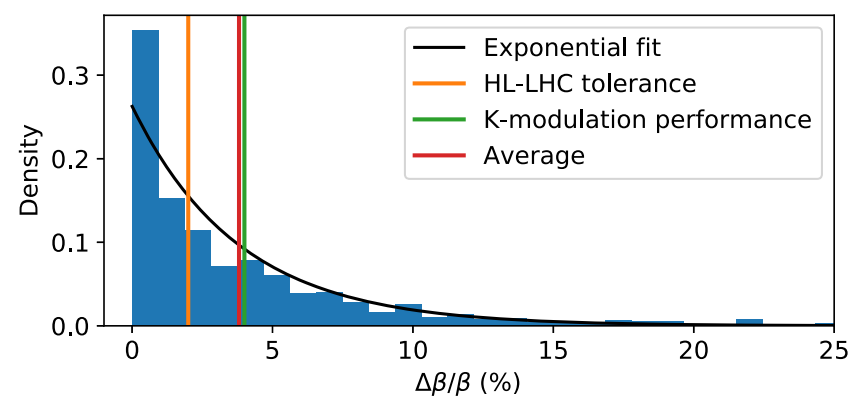

FIG. 5. Remaining $\beta$-beating in IP1 and IP5 in the horizontal and vertical planes for 100 simulation of triplet and Q4-5 errors after the automatic local corrections, for a precision in $\mathrm{K}$ modulation compatible with $4 \%$ precision in the measurement of $\beta$-beating. The standard deviation of the distribution is $3.8 \%$, very close to the precision in K-modulation.

To simulate the K-modulation measurement, the matching algorithm is also provided with the value of the $\beta^{*}$ with an uncertainty of $4 \%$, corresponding to a tune uncertainty of $2.5 \times 10^{-5}$ as shown in Fig. 4 .

This value of the tune uncertainty is optimistic as it assumes an upgrade of the arcs power supplies that is not in the HL-LHC baseline, as detailed in [9]. However, the target of the algorithm is to correct down to this $\beta^{*}$ uncertainty level, therefore this assumption does not bias the results.

Figure 5 shows the remaining $\beta^{*}$-beating in IP1 and IP5, after applying the automatic correction in each seed. The standard deviation of the resulting distribution is $\sigma=3.8 \%$, very close to the given uncertainty of K-modulation, which indicates that the automatic matching routine successfully corrects the $\beta^{*}$-beating down to the uncertainty of the $\beta^{*}$ measurement.

Figure 6 shows the relative difference between the error in each quadrupole of the triplet and the correction found by the matching routine. The correction strength stays in general on the level of the magnetic errors $\left(10^{-3}\right)$ but raises to about $5 \times 10^{-3}$ in r.m.s. in the $\mathrm{Q} 1 \mathrm{~s}$, probably to compensate for the $2 \mathrm{~mm}$ misalignment and Q4 and Q5 errors.

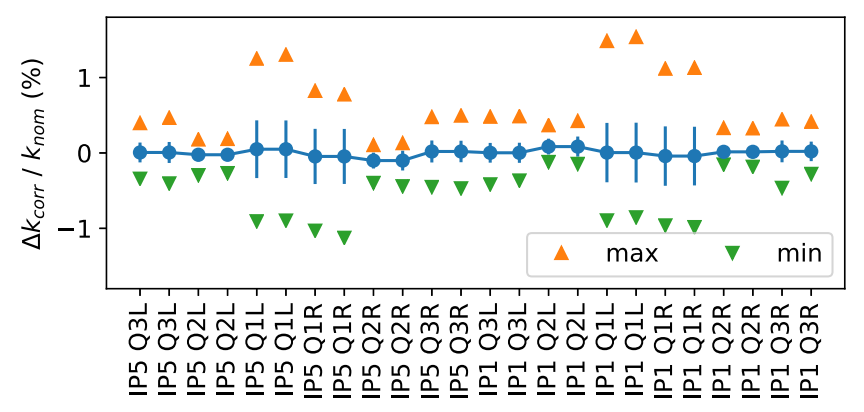

FIG. 6. Relative difference between the magnetic errors of each seed and the correction implemented in each triplet quadrupole. 


\section{Triplet tilt tolerances in the HL-LHC}

A tilted quadrupole interacts with the beam as a straight quadrupole plus a skew quadrupolar component. This skew component will have an effect in the $f_{1001}$ resonance driving term given by [17]:

$f(s)_{1001}=\frac{-1}{4\left(1-e^{2 \pi i\left(Q_{x}-Q_{y}\right)}\right)} \sum_{l} k_{l} \sqrt{\beta_{x}^{l} \beta_{y}^{l}} e^{i\left(\Delta \phi_{x}^{s l}-\Delta \phi_{y}^{s l}\right)}$

where $k_{l}$ is the $l^{\text {th }}$ skew quadrupole integrated strength, $\beta_{x, y}^{l}$ are the $\beta$-functions at the position of the $l$ th skew quadrupole, $\Delta \phi_{x, y}^{s l}$ are the phase-advances between the measurement point and the $l$ th skew quadrupole and $Q_{x, y}$ are the horizontal and vertical tunes. It can be seen how due to the large $\beta$-function across the HL-LHC triplet shown in Fig. 2, tilts of the triplet quadrupoles have the potential to be massive contributors to the magnitude of the $f_{1001}$ term and therefore, to the coupling in the region and in the whole machine if not properly compensated locally. In HL-LHC, a skew quadrupole corrector will be installed in each side of IP1 and IP5 (Fig. 2 top schematic) to cancel out the local coupling produced by the triplet.

In order to define the required strength of these correctors, we performed automatic corrections of 500 simulation of the HL-LHC triplet expected tilts. On each seed, all the quadrupoles of the triplet and Q4-5 were given a random tilt within $\pm 1 \mathrm{mrad}$ and $\pm 2 \mathrm{mrad}$ (uniformly distributed). Also, a random choice among 60 seeds of sextupolar to dodecapolar normal and skew multipolar errors (b3, a3, b4, $\mathrm{a} 4, \mathrm{~b} 5, \mathrm{a} 5$, b6 and a6) was given to each seed. The simulations were performed at $\beta^{*}=30 / 7.5 \mathrm{~cm}$ flat optics (worst case scenario) with a crossing angle of $250 \mu \mathrm{rad}$.

Figure 7 shows the resulting strengths of the skew quadrupoles as calculated by the automatic matching routine for each of the 500 seeds. The measurable traditionally used to quantify the global coupling is the $\Delta Q_{\text {min }}$ i.e. the minimum tune difference between the horizontal and vertical tunes produced by coupling [18]. For these simulations, after the local coupling corrections, the uncorrected coupling that leaks from the IR produces a $\Delta Q_{\text {min }}$ below $10^{-3}$ for all seeds.

The solid lines of Fig. 7 show the original design strength of the HL-LHC skew quadrupole correctors: $10^{-3} \mathrm{~m}^{-2}$. Having such a large margin available, if the tilt is guaranteed to stay within $\pm 1 \mathrm{mrad}$, which can be assured using the remote alignment system [19], the design strength for the correctors can be reduced to $0.7 \times 10^{-3} \mathrm{~m}^{-2}$ (dashed lines) while still allowing a factor 2 margin in case one of the correctors stops functioning. The resulting strengths of the right and left correctors in each seed show a tendency to lay across the diagonal, namely, to set both correctors to a similar strength.

Quadrupoles can also present local tilts along their length (waviness). Monte-Carlo simulations of this effect have also been performed, applying local tilts to 10 thick slices

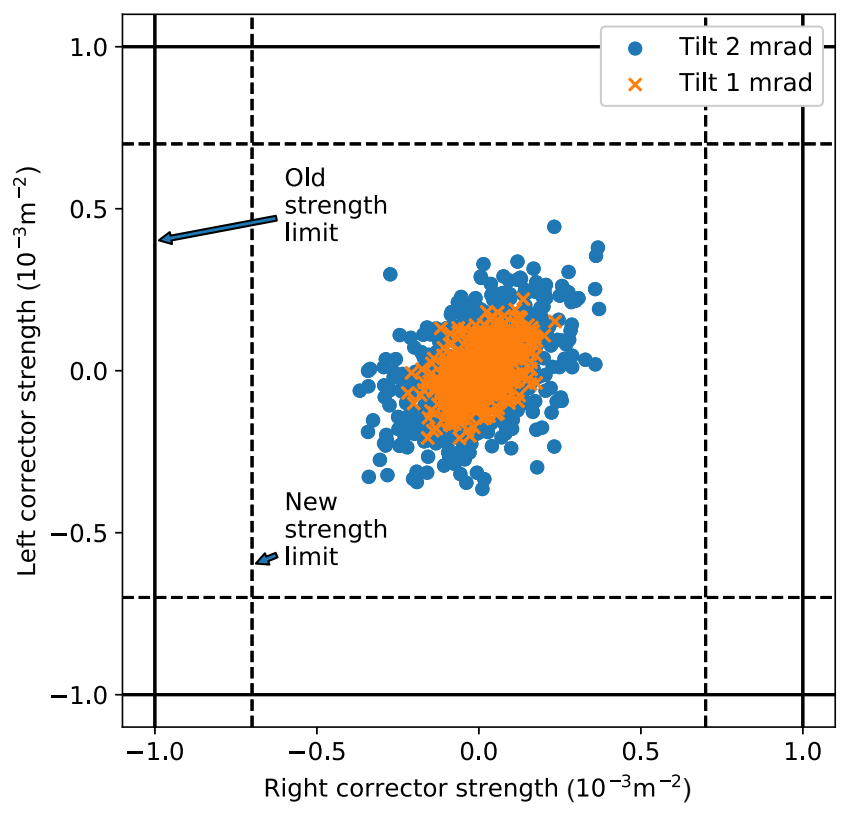

FIG. 7. Required strength of the skew quadrupole correctors in IR1 to cancel the coupling produced by 500 seeds of random tilts of $\pm 1 \mathrm{mrad}$ and $\pm 2 \mathrm{mrad}$ in Q4-5 and the triplet quadrupoles. The solid lines show the old specification for the strength limit of the correctors and the dashed lines the new specification set by this work.

of each quadrupole of the triplet, concluding that up to \pm 5 mrad uniformly distributed waviness has a negligible effect when compared with the average tilt of the magnet. This means that a \pm 5 mrad waviness is tolerated if the average tilt is controlled within $\pm 1 \mathrm{mrad}$.

These simulation results show, in conclusion, that the automatic matching routine is able to correct local optics to the $\beta^{*}$ measurement accuracy. Therefore, the limiting factor for HL-LHC to deliver the design luminosity and to keep the luminosity imbalance within tolerable levels, is the accuracy of the measurement of the $\beta^{*}$. In following sections we will describe the problems of currently used techniques and possible alternatives for HL-LHC. The matching routine also succeeded defining the required strength of the skew quadrupole correctors in IR1 and IR5, allowing a reduction of these correctors strengths, reducing the construction cost of HL-LHC.

\section{K-MODULATION ISSUES}

$\mathrm{K}$-modulation has been the technique used to measure and control the LHC $\beta^{*}$ during Run 2 [2]. However, for

TABLE I. Comparison of the average over 5 measurements of the vertical waist displacements measured by K-modulation and the waist obtained using the luminosity scans.

\begin{tabular}{lcc}
\hline \hline Waist $[\mathrm{cm}]$ & From K-mod & From luminosity scan \\
\hline Beam 1 & $-5 \pm 3$ & $-8.1 \pm 1.3$ \\
Beam 2 & $4 \pm 2$ & $0.29 \pm 0.09$ \\
\hline \hline
\end{tabular}


TABLE II. K-modulation measurements before (No correction) and after (Correction) of the $\beta^{*}$, waist and deviation from the model of the $\phi_{\mathrm{IP}}$ both from K-modulation [Eq. (3)] and from excited beam BPM recordings (kicks) at flat optics $\beta^{*}=60 / 15 \mathrm{~cm}$. The problem appeared in IP5 in the vertical plane in 2017 and in the horizontal plane of IP1 in 2018, both on Beam 1 . The design $\beta^{*}$ is $60 \mathrm{~cm}$ in the vertical plane of IP5 and the horizontal plane of IP1 (the flat plane is swapped in IP1 and IP5).

\begin{tabular}{lcccc}
\hline \hline & $\beta^{*}[\mathrm{~m}]$ & Waist $[\mathrm{m}]$ & $\Delta \phi_{\mathrm{K} \text {-mod }}\left[2 \pi 10^{-4}\right]$ & $\Delta \phi_{\text {kicks }}\left[2 \pi 10^{-4}\right]$ \\
\hline 2017 No correction & $1.45 \pm 0.05$ & $0.72 \pm 0.03$ & $-104 \pm 8$ & $-4 \pm 13$ \\
2017 Correction & $1.17 \pm 0.06$ & $0.57 \pm 0.03$ & $-79 \pm 10$ & $-4 \pm 12$ \\
2018 No correction & $0.83 \pm 0.03$ & $0.32 \pm 0.03$ & $-26 \pm 4$ & $-6 \pm 7$ \\
\hline \hline
\end{tabular}

low- $\beta^{*}$ optics, this technique is starting to show its limitations. In Table I the average and r.m.s. of beam waist measurements performed at $\beta^{*}=30 \mathrm{~cm}$ (the operational optics of 2018) is presented, showing a large spread over several measurements. During studies of flat optics with $\beta^{*}=60 / 15 \mathrm{~cm}$, this technique showed systematic unreliable measurements of the $\beta^{*}$ since K-modulation is validated against phase measurements with the $\mathrm{AC}$ dipole as explored in the following.

The formula that describes the $\beta$-function across the optics drift around the IP follows

$$
\beta(s)=\beta_{w}+\frac{(s-w)^{2}}{\beta_{w}},
$$

with $\beta(0)=\beta^{*}$ the $\beta$-function at the IP and $w$ the waist position with respect to the IP, i.e., the point where $\beta(w)=\beta_{w}$, the minimum $\beta$-function in the drift.

The phase-advance between the start and the end of this optics drift follows

$$
\phi_{\mathrm{IP}}=\arctan \left(\frac{L^{*}-w}{\beta_{w}}\right)+\arctan \left(\frac{L^{*}+w}{\beta_{w}}\right),
$$

being $L^{*}$ the distance between the BPM and the IP, assuming that the BPM is upstream of the first triplet quadrupole when moving from the IP. As K-modulation provides the $\beta_{w}$ and waist position, this $\phi_{\mathrm{IP}}$ can be compared with the $\phi_{\text {IP }}$ measured using BPM turn-by-turn data of beam excitations with the ac dipole.

Table II shows K-modulation measurements of Beam 1 performed at $\beta^{*}=60 / 15 \mathrm{~cm}$ optics for the vertical plane of IP5 in 2017 [20] and for the horizontal plane of IP1 in 2018. In both cases, the design $\beta^{*}$ is $60 \mathrm{~cm}$. The measurements show a not too large error and, in principle, should be trusted. By contrast, the table also shows the deviation of $\phi_{\mathrm{IP}}$ with respect to the model for both K-modulation computed using the formula above and from standard BPM measurements on beam excitations with the ac dipole. The phase measured from K-modulation is not compatible with that measured by BPMs for these 3 cases. The reason for this disagreement is not clear, but shows that K-modulation can give unreliable results in certain conditions.
One option to increase the accuracy of K-modulation could be to increase the strength of the modulation allowing larger tune changes. The limit of the change in the tune produced by K-modulation is set to be $\frac{1}{3}\left(Q_{x}-Q_{y}\right)=10^{-2}$ [8] at injection tunes, to avoid hitting the coupling stopband. In principle, the LHC tune feedback system [21] could be used to compensate the change on the tune removing this limit and allowing for stronger modulations. In Fig. 8, an attempt to perform K-modulation with the tune feedback active is shown. It should be noted that the modulation of the tunes is not totally removed, it remains an oscillation of about $10^{-3}$. Also in the vertical tune of Beam 2 a delay time in the reaction of the system can be observed. These technical difficulties would make this approach unfeasible with the current system. Moreover,

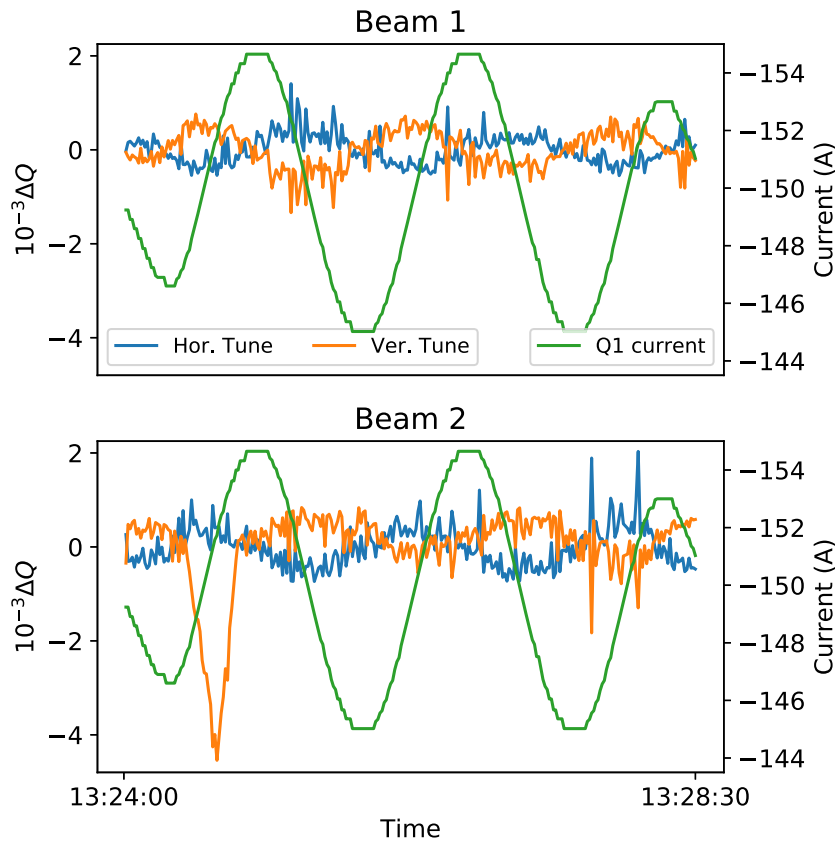

FIG. 8. K-modulation performed during ATS optics MD studies in 2016 with the tune feedback system enabled. A residual oscillation of about $10^{-3}$ remains uncompensated. Also the system reacted too late compensating the vertical tune in Beam 2, as can be seen in the start of the orange line in the bottom plot. 


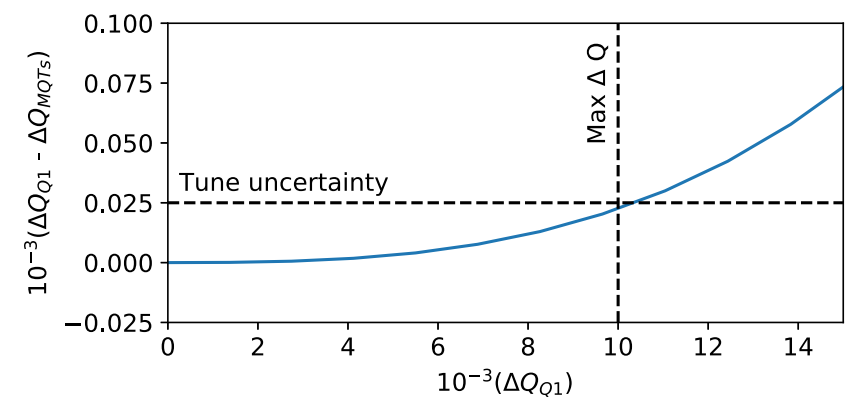

FIG. 9. Simulation of a K-modulation using the tune feedback. Here $\Delta \mathrm{Q}_{Q 1}$ is the change of tune produced by the trim of the Q1 and $\Delta \mathrm{Q}_{\mathrm{MQT}}$ the change of tune produced by the arcs tuning quadrupoles to compensate the Q1 effect. The horizontal line shows the tune measurement uncertainty of $2.5 \times 10^{-5}$. When the curve is above this line the cross-talk between Q1 and arcs tuning quadrupoles is dominating the error of the K-modulation measurement. The vertical line shows the maximum allowed change of tune in the absence of tune feedback.

Fig. 9 shows a simulation of K-modulation with tune feedback using the arcs tuning quadrupoles correctors (MQTs) to keep a constant tune. It shows the difference in the tune change from Q1 and the tune change inferred from the feedback quadrupoles (MQTs) versus the Q1 tune change, demonstrating how the cross talk between the $\beta$ beating wave produced by the modulation of Q1 and the MQTs dominates over the error caused by the tune uncertainty for modulations higher than $10^{-2}$. Therefore, K-modulation with the tune feedback presents significant difficulties both on the technical and analytical sides and it is discarded for the future.

Due to the first unreliable observations of K-modulation during Run 2 and the failure to operate it with the tune feedback to increase its accuracy, it will be essential to have alternative techniques to K-modulation for the Run 3 of the LHC and in view of HL-LHC where the $\beta^{*}$ will be even lower.

\section{IV. $\beta_{w}$ FROM PHASE-ADVANCE}

The need to squeeze the beam to very low- $\beta^{*}$ pushes the $\beta$-function in the IR region to very large values and therefore, the betatron phase-advance becomes so small that is barely measurable with the standard BPM resolution in the LHC. Nevertheless, an expression to obtain the $\beta_{w}$ from the $\phi_{\mathrm{IP}}$ can be derived and rely on BPMs with higher precision in the measurement of the phase-advance. Expanding Eq. (3) around $w=0$ up to second order in $w$ :

$$
\phi_{\mathrm{IP}}=2 \arctan \left(\frac{L^{*}}{\beta_{w}}\right)-\frac{2 w^{2} \beta_{w} L^{*}}{\left(\beta_{w}^{2}+L^{* 2}\right)^{2}}+O\left(w^{4}\right),
$$

shows that, to the lowest order, $\phi_{\mathrm{IP}}$ decreases with the square of the waist position and we can approximate $\beta_{w}$ from $\phi_{\mathrm{IP}}$ as:

$$
\beta_{w}=\frac{L^{*}}{\tan \left(\frac{\phi_{\mathrm{IP}}}{2}\right)}-\frac{w^{2} \beta_{w} L^{* 2}}{\left(\beta_{w}^{2}+L^{* 2}\right)^{2} \sin \left(\frac{\phi_{\mathrm{IP}}}{2}\right)}+O\left(w^{4}\right) .
$$

Assuming the term $\left(\beta_{w}^{2}+L^{* 2}\right)^{2}$ is dominated by $L^{* 4}$ and that $\phi_{\mathrm{IP}} \approx \pi$ we get:

$$
\beta_{w} \approx \frac{L^{*}}{\tan \left(\frac{\phi_{\mathrm{IP}}}{2}\right)}-\frac{w^{2} \beta_{w}^{2}}{L^{* 2} \beta_{w}} .
$$

Isolating the function of $\phi_{\mathrm{IP}}$ that we can measure and defining $w_{\text {eff }}=\frac{w \beta_{w}}{L^{*}}$ :

$$
\frac{L^{*}}{\tan \left(\frac{\phi_{\mathrm{IP}}}{2}\right)} \approx \beta_{w}+\frac{\left(\frac{w \beta_{w}}{L^{*}}\right)^{2}}{\beta_{w}}=\beta_{w}+\frac{w_{\mathrm{eff}}^{2}}{\beta_{w}} .
$$

A comparison with Eq. (2) shows that the observable being measured is the $\beta\left(w+w_{\text {eff }}\right)$ at a distance $w_{\text {eff }}$ from the actual waist. This disturbance is due to the fact that the waist is displacing the minimum of the parabola from the center of the two measuring BPMs.

For the measurement of the $\beta_{w}$ to stay accurate, it has to hold that:

$$
\frac{\left(\frac{w \beta_{w}}{L^{*}}\right)^{2}}{\beta_{w}} \ll \beta_{w} \rightarrow w \ll L^{*} .
$$

The $L^{*}$ in the LHC is of the order of tens of meters and the typical waist shifts are several centimetres.

In Fig. 10 the behavior of $\phi_{\mathrm{IP}}$ is shown versus $\beta_{w}$ for a $\pm 50 \%$ range from $15 \mathrm{~cm}$ and versus the waist position for a range of $\pm 25 \mathrm{~cm}$. The effect of the waist displacement on $\phi_{\mathrm{IP}}$ is four orders of magnitude lower than that of the $\beta_{w}$, becoming smaller for lower $\beta_{w}$.

Ignoring the influence of the waist displacement we obtain a simpler formula:

$$
\beta_{w} \approx \frac{L^{*}}{\tan \left(\frac{\phi_{\mathrm{IP}}}{2}\right)} .
$$

All the approximations made from Eq. (5) to Eq. (9) produce a maximum error in $\beta_{w}$ for the ranges of $\beta_{w}$ and waist shown in Fig. 10 of about $0.01 \%$.

Defining $\sigma\left(\beta_{w}\right)$ and $\sigma\left(\phi_{\mathrm{IP}}\right)$ as the uncertainties of the measurements of $\beta_{w}$ and $\phi_{\mathrm{IP}}$ respectively, we obtain:

$$
\sigma\left(\beta_{w}\right) \approx \frac{L^{*}}{\left|\cos \left(\phi_{\mathrm{IP}}\right)-1\right|} \sigma\left(\phi_{\mathrm{IP}}\right),
$$

or in relative terms to $\beta_{w}$ :

$$
\frac{\sigma\left(\beta_{w}\right)}{\beta_{w}}=\frac{\sigma\left(\phi_{\mathrm{IP}}\right)}{\left|\sin \phi_{\mathrm{IP}}\right|} .
$$

This $\phi_{\mathrm{IP}}$ is typically close to $\pi$. For instance, in the case of $\beta^{*}=50 \mathrm{~cm}$ ion optics the design $\sin \left(\phi_{\mathrm{IP}}\right)$ is about 

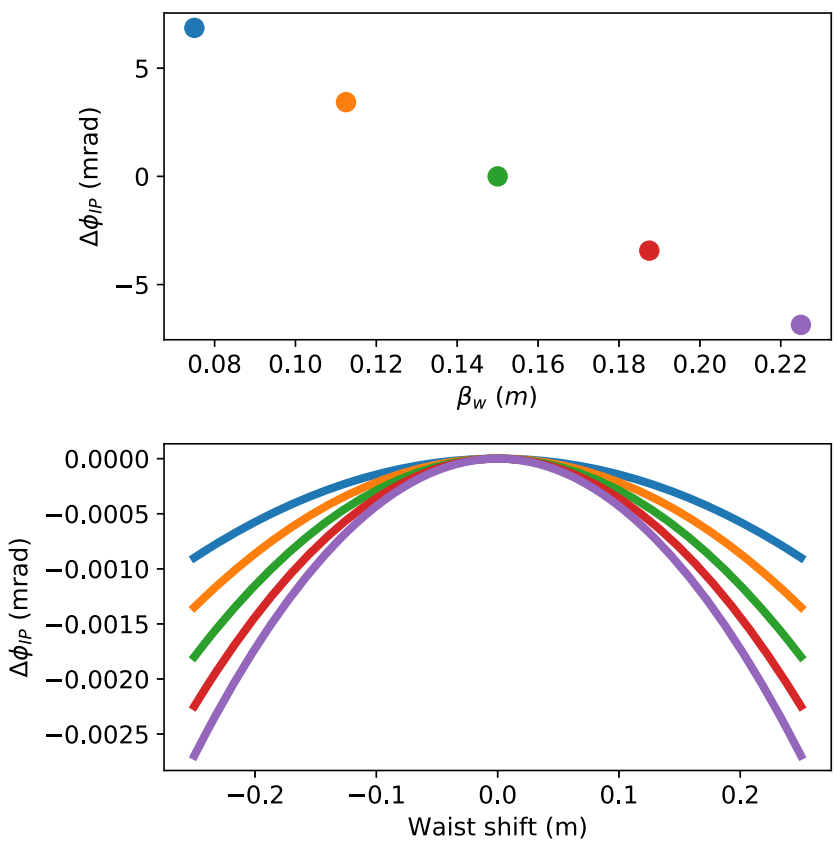

FIG. 10. Behaviour of the phase-advance change across the IP optics drift with changing $\beta_{w}$ (top) and changing waist shift (bottom). As the change with waist shift depends significantly on the $\beta_{w}$ the phase deviation with changing waist shift is shown for the different $\beta_{w}$ in the upper plot, with corresponding colors.

$5 \times 10^{-2}$. To obtain a $2 \%$ precision in $\sigma\left(\beta_{w}\right) / \beta_{w}$ a precision of $\approx 2 \times 10^{-2} \times 5 \times 10^{-2}=10^{-3} \mathrm{rad}$ in the measurement of the phase is necessary.

\section{A. The DOROS BPM system}

The diode orbit and oscillation (DOROS) BPM system $[22,23]$ is installed in the LHC tertiary collimators jaws and next to the IPs. This opens the possibility of measuring the $\beta_{w}$ from phase measurements coming from this system using Eq. (9). It provides better phase resolution than standard BPMs and can be triggered together with them.

The DOROS BPM system was triggered during the ion run commissioning of 2018 in the LHC, with $50 \mathrm{~cm} \beta^{*}$ optics. A total of 6 acquisitions of the standard BPM system and 8 acquisitions of the DOROS system were triggered. Using these acquisitions, a standard deviation on the phase advance across the IR of $0.3 \mathrm{mrad}$ was found using the DOROS system and $1.1 \mathrm{mrad}$ using the standard BPM system, about a factor 4 larger. Applying Eq. (9) on this measurement of the phase advance results $\beta_{w}=0.56 \pm$ 0.01 for the normal LHC BPM system and $\beta_{w}=0.544 \pm$ 0.003 for the DOROS BPM system.

In Fig. 11 the previous measurement of the precision of the phase advance across the IR is extrapolated to smaller $\beta^{*}$ showing how the DOROS system provides a $\beta_{w}$ measurement below the required error for $2 \%$ precision for all the optics in the range from $50 \mathrm{~cm}$ to $7.5 \mathrm{~cm} \beta_{w}$, positioning the phase-advance measurement with DOROS

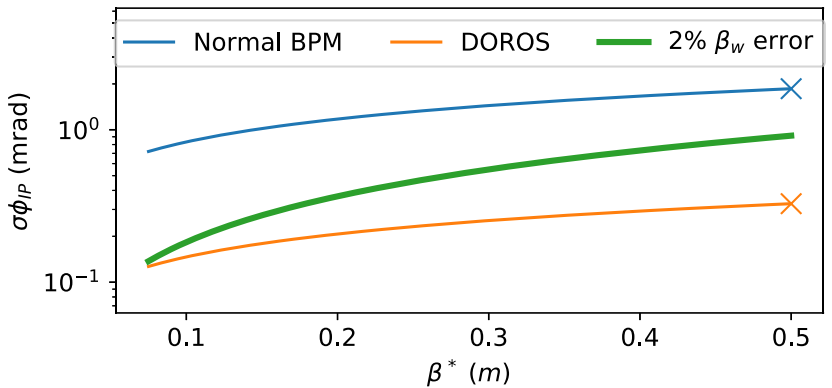

FIG. 11. Expected measurement error of the standard and DOROS BPM systems taking as reference a measurement at $50 \mathrm{~cm} \beta^{*}$. The green line shows the required phase measurement error to achieve a $2 \%$ error in $\beta_{w}$.

BPMs as a good alternative to K-modulation to measure $\beta_{w}$. However, as it was shown before, it does not give information about the actual waist shift which can strongly impact the $\beta^{*}$. In the next section, the use of luminosity scans to measure the waist shift is proposed.

\section{LUMINOSITY SCANS}

An alternative technique to measure waist shifts is to design a set of quadrupole trims that displace the beam waist. Applying such knobs step by step, the beam waist changes linearly and $\beta^{*}$ quadratically as shown in Fig. 12. Monitoring the luminosity the position of the beam waist can be interpolated.

Luminosity waist scans have been performed in other colliders as SuperKEKB [24,25], however the particularities of the LHC make this technique significantly more challenging. In the LHC, machine protection constraints force us to keep the $\beta$-beating in the machine within safe values during the scans, which poses a strong constraint in the design of the waist shift knobs and their operational range. The luminosity changes for the design waist shift of about $10 \mathrm{~cm}$ are in the order of about $1 \%$, significantly

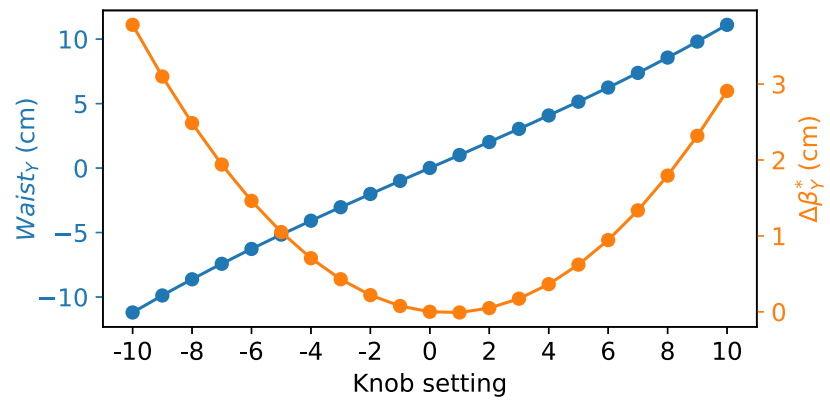

FIG. 12. Simulated effect on the design model with $\beta^{*}=30 \mathrm{~cm}$ optics of one of the waist displacement knobs experimentally tested in the LHC. The blue line represents the waist displacement and is almost completely linear with the knob value. The orange line shows the $\beta^{*}$ following the parabola of Eq. (2) as the waist is displaced. The minimum of the parabola is not at the zero knob setting because the design optics includes a small waist. 


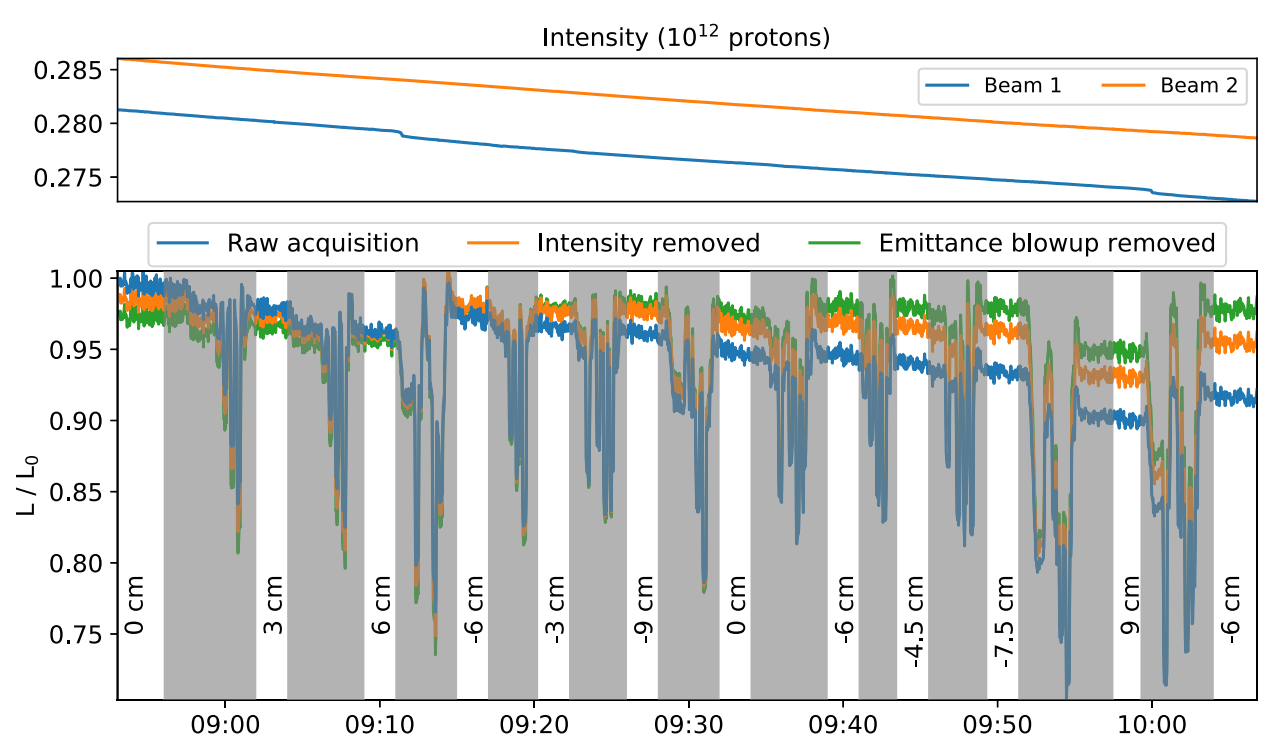

FIG. 13. Instantaneous luminosity (relative to the first raw measurement) recorded by ATLAS during the first waist scan. In blue the raw luminosity as given by ATLAS and the orange line is normalized to the beams intensity. The green line has the emittance blowup decay removed and is the one used to fit the beam waist parabola. The parts removed from the analysis are greyed out.

smaller than in other colliders, challenging the data acquisition and the analysis accuracy as described below.

The luminosity measured in each IP follows the formula:

$$
L=\frac{N_{b} f N_{1} N_{2} S}{2 \pi \sqrt{\epsilon_{x 1} \beta_{x 1}^{*}+\epsilon_{x 2} \beta_{x 2}^{*}} \sqrt{\epsilon_{y 1} \beta_{y 1}^{*}+\epsilon_{y 2} \beta_{y 2}^{*}}},
$$

where $N_{b}$ is the number of bunches in the machine, $N_{1,2}$ are the number of particles in each bunch, $\epsilon_{x 1, y 1, x 2, y 2}$ the emittances in each plane and beam and $f$ the beam revolution frequency. $\mathrm{S}$ is the beam shape parameter that can be approximated (ignoring the hourglass effect) as [26]:

$$
S=\frac{1}{\sqrt{1+\left(\frac{\sigma_{s}}{\sigma_{x}} \frac{\theta}{2}\right)^{2}}}=\frac{1}{\sqrt{1+\phi_{p}^{2}}},
$$

with $\theta$ the crossing angle at the IP, $\sigma_{s}$ the longitudinal beam size, and $\phi_{p}$ is the so-called Piwinski angle.

For the case of a waist knob designed to displace the horizontal waist of Beam 1, assuming all emittances are equal and normalising to the luminosity at the waist $L_{w}=L(w)$, the dependency of the luminosity on the $\beta^{*}$ goes as

$$
\frac{L(s)}{L_{w}}=\sqrt{\frac{\beta_{w}\left(1+\frac{\phi_{p}^{2}}{\sqrt{2}}\right)+\beta_{x 2}^{*}}{\beta_{w}\left(1+\frac{\phi_{p}^{2}}{\sqrt{2}}\right)+\beta_{x 2}^{*}+\frac{(s-w)^{2}}{\beta_{w}}}}
$$

with $s$ the value of the waist displacement knob, $w$ the actual waist present in the machine in Beam $1, \beta_{w}$ the beta on the waist of Beam 1 and $\beta_{x 2}^{*}$ the $\beta^{*}$ of Beam 2 .
The full luminosity equation can then be expanded for small $(s-w)$ as

$$
\frac{L(s)}{L_{w}}=1-\frac{(s-w)^{2}}{2 \beta_{w}\left[\beta_{w}\left(1+\frac{\phi_{p}^{2}}{\sqrt{2}}\right)+\beta_{x 2}^{*}\right]}+O\left[(s-w)^{4}\right] .
$$

The right-hand side (r.h.s.) of this equation has the shape of a parabola and the values of the waist position $w$ and the denominator can be extracted from a fit of the luminosity measurement against several values of the waist scan knob $s$. The denominator contains the information of the $\beta_{w}$, but the presence of the Piwinski angle means that a very precise measurement of the crossing angles and longitudinal beam profile is required to obtain a good measurement of the $\beta_{w}$. The typical resolution of both $\sigma_{s}$ and the crossing angle is about $10 \%$ [27] and therefore this method cannot be used to obtain a precise measurement of the $\beta_{w}$. To obtain a precise

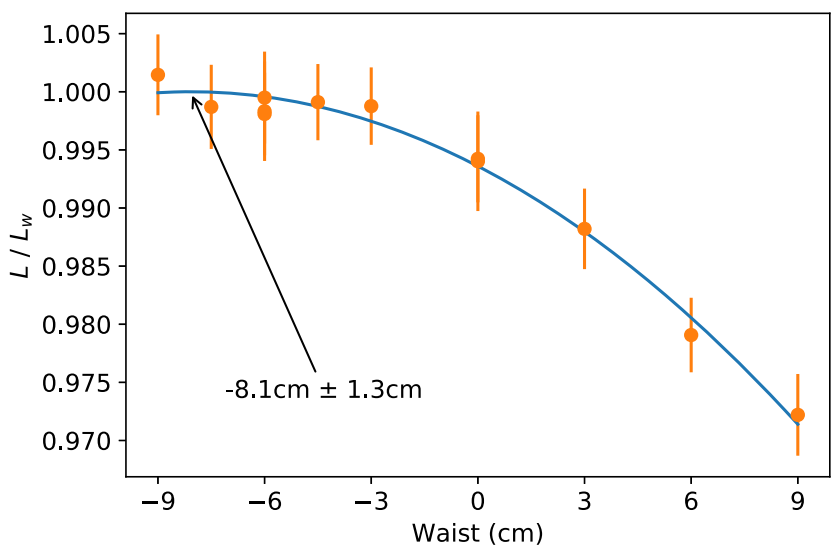

FIG. 14. Luminosity scan of Beam 1 on the vertical plane. 


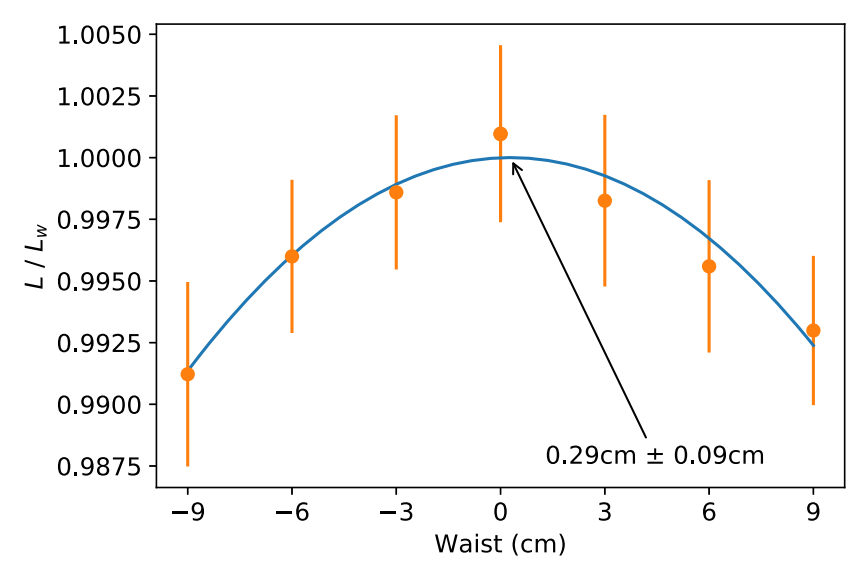

FIG. 15. Luminosity scan of Beam 2 on the vertical plane.

measurement of the $\beta_{w}$ the phase-advance from DOROS BPMs can be used as described in Sec. IV.

\section{A. Experimental validation}

Waist scans have been successfully performed in the LHC during a machine study experiment the 16th of September of 2018 with nominal optics, $\beta^{*}=30 \mathrm{~cm}$. Using MAD-X, two knobs were designed to shift the vertical waist of Beam 1 (Fig. 12) and 2 at IP1.

In Fig. 13, the instantaneous luminosity acquisition from ATLAS for the first of these scans is shown. The periods when luminosity optimization was being performed (dips in the signals) or when the knobs were being trimmed were discarded from the analysis and are shown greyed out. There is a decay in the raw luminosity signal (blue line) caused by the beam burn-off produced by the collisions. This means that in Eq. (12) the terms $N_{1,2}$ are timedependent. To remove this time dependency, the luminosity is divided by the measured $N_{1}(t) N_{2}(t)$ during the experiment, resulting in the orange line of Fig. 13. After this operation, the signal still has a noticeable decreasing trend, which is probably due to emittance blowup during the experiment. As the points with the same knob settings should have the same luminosity value, additional effects like this emittance blowup can be removed by fitting a line over measurements with the same settings and rescaling the luminosity value to the found fit. In this case a line was fitted over two measurements when the knob waist was set to zero.

After postprocessing the data as described, the parameters of Eq. (15) can be fitted to the scaled luminosity versus knob setting. In Fig. 14, the scaled luminosity versus waist knob setting from -9 to $9 \mathrm{~cm}$ is shown, together with the parabolic fit. The measured vertical waist shift is $-8.1 \pm 1.3 \mathrm{~cm}$ in Beam 1 and $0.29 \pm 0.09 \mathrm{~cm}$ in Beam 2 as shown in Fig. 15. Table I shows a comparison between the average and r.m.s. over 5 measurements of the waist position using K-modulation performed during 2018 and the results of the luminosity scans. It should be noted that the luminosity scans measurements have a significantly lower uncertainty. This first experimental results are promising, but to allow their use in operation the luminosity scans will have to be streamlined and their reproducibility over time studied with further scans.

Altogether, these are the first successful waist measurement using luminosity scans in the LHC, a machine with strong machine protection constraints. These results open the gate to complement K-modulation with a combination of IP phase advance measurements and luminosity scans, allowing a more precise determination of the $\beta^{*}$, which as was described before, will be of crucial importance to guarantee the performance of the HL-LHC.

\section{LOCAL ARC CORRECTIONS WITH ORBIT BUMPS}

Number of experimental tests were performed with flat optics i.e. different horizontal and vertical $\beta^{*}$ of $60 / 15 \mathrm{~cm}$ $[20,28,29]$. The ambitious optics configurations are

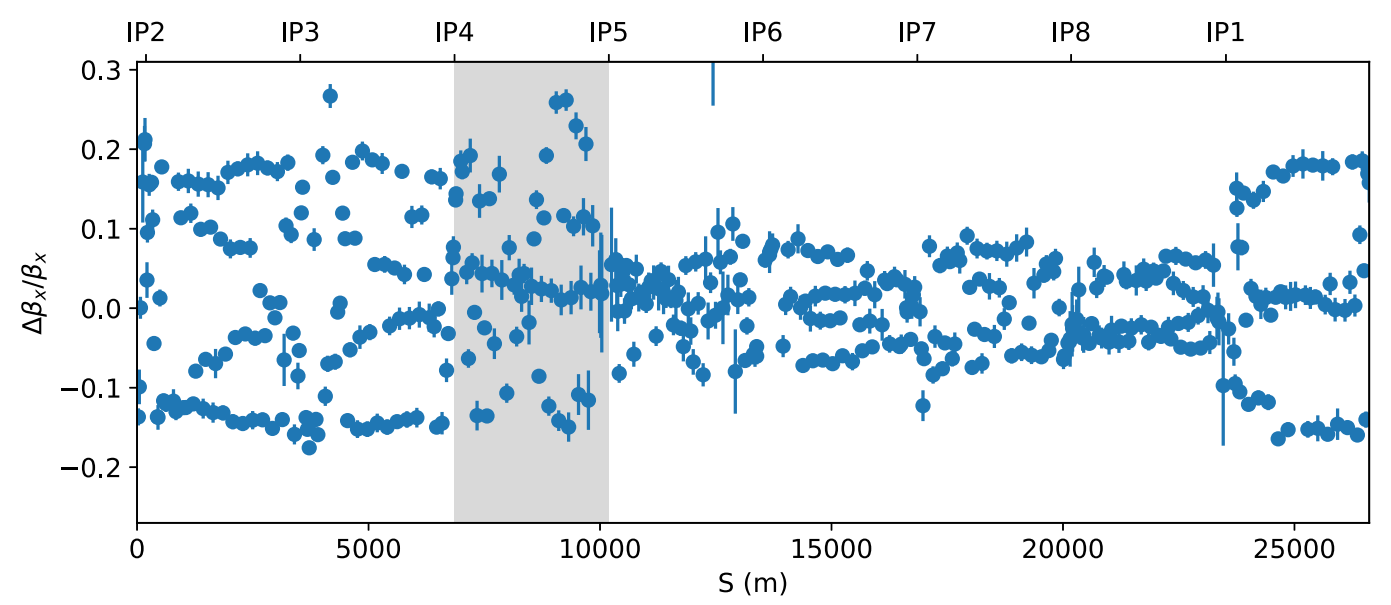

FIG. 16. Horizontal $\beta$-beating measurement at flat $60 / 15 \mathrm{~cm}$ optics with local and global corrections applied, performed during the 2017 flat optics MD. The grey area shows the arc 45. In this area the corrections were unable to bring the $\beta$-beating below $24 \%$. 

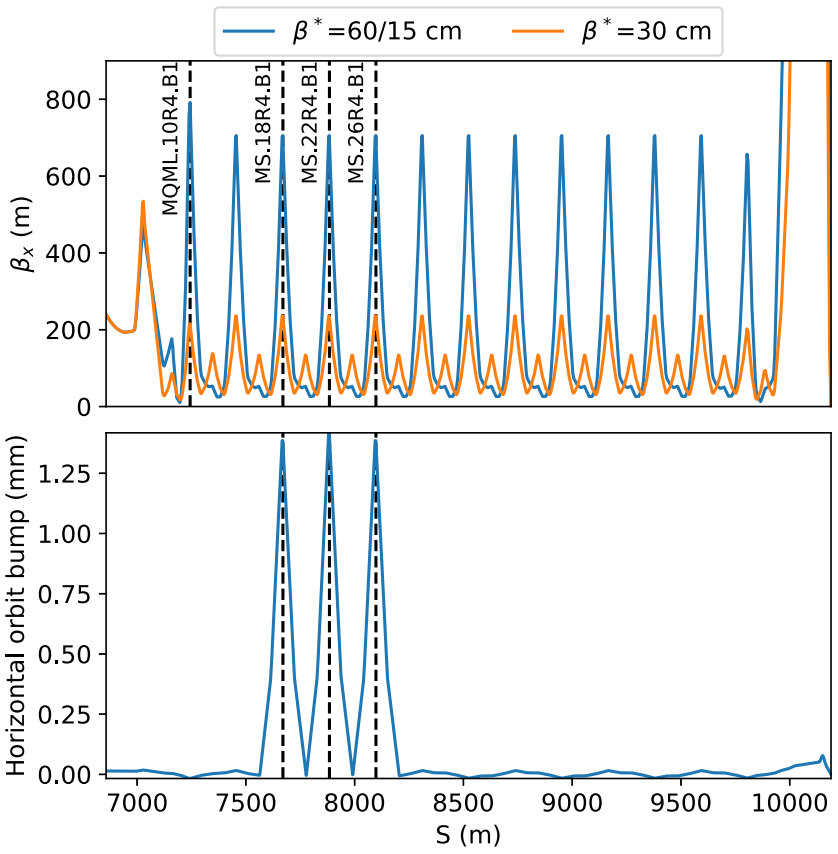

FIG. 17. The top plot shows the design horizontal $\beta$-function from IP4 to IP5 for $\beta^{*}=60 / 15 \mathrm{~cm}$ flat optics and for $\beta^{*}=30 \mathrm{~cm}$, the nominal 2018 optics. The ATS optics scheme increases the $\beta$-function in this arc by a factor of 4 . The bottom plot shows the horizontal orbit bump implemented in the sextupoles to correct the optics via feed-down.

achieved using the ATS scheme. The ATS scheme will also be used in the HL-LHC where it is expected to reach a $\beta^{*}=30 / 7.5 \mathrm{~cm}$ in flat optics [14].

During the $2017 \mathrm{MD}$ on flat optics with $\beta^{*}=60 / 15 \mathrm{~cm}$ [20], it was not possible to bring the $\beta$-beating in arc 45 below $24 \%$ after local and global corrections, as shown in Fig. 16, highlighting in grey the problematic arc. $20 \%$ peak $\beta$-beating is considered the upper limit to safely operate the LHC and in 2016 with $\beta^{*}=40 \mathrm{~cm}$ the rms $\beta$-beating in the machine was brought down to the $2 \%$ r.m.s. level [2].
TABLE III. Trim settings implemented to correct arc 45 at $\beta^{*}=60 / 15 \mathrm{~cm}$. It combines a trim on the Q10 on the right side of IR4 (MQML.10R4.B1) with an orbit bump created using the dipoles shown in the table $(\mathrm{MCBH})$ that peaks in the main sextupoles MS.18R4.B1, MS.22R4.B1 and MS.26R4.B1 reaching $1.5 \mathrm{~mm}$.

\begin{tabular}{llc}
\hline \hline Magnet & \multicolumn{1}{c}{ Circuit } & Trim $\left[10^{-6}\right]$ \\
\hline MQML.10R4.B1 & RQ10.R4B1 & $20 \mathrm{~m}^{-2}$ \\
MCBH.16R4.B1 & RCBH16.R4B1 & $8 \mathrm{rad}$ \\
MCBH.20R4.B1 & RCBH20.R4B1 & $16 \mathrm{rad}$ \\
MCBH.24R4.B1 & RCBH24.R4B1 & $16 \mathrm{rad}$ \\
MCBH.28R4.B1 & RCBH28.R4B1 & $8 \mathrm{rad}$ \\
\hline \hline
\end{tabular}

Using the segment-by-segment technique on arc 45 , this error was identified as a local error in this arc enhanced by the high design $\beta$-function of the ATS optics scheme. The peak $\beta$-beating produced by a gradient error in arc 45 might be enhanced up to a factor 4 when comparing $30 \mathrm{~cm}$ and flat $60 / 15 \mathrm{~cm}$ optics, as shown in Fig. 17 (top).

In that region of the accelerator, there are no individually-powered quadrupoles to correct the error, which explains the difficulties found in 2017 to correct arc 45 using global corrections. However, it is possible to produce quadrupolar fields via feed-down from sextupoles by traversing them with a nonzero orbit.

In Fig. 18 the results from segment-by-segment of the phase deviation from the local model of arc 45 under $60 / 15 \mathrm{~cm}$ flat optics that was measured on 2017 is shown in blue. A solution to match this phase measurement was found to be a $5.2 \mathrm{~mm}$ horizontal orbit bump with maximum in the MS.26R4.B1 sextupole. To avoid this large bump, the correction was spread to the MS.18R4.B1 and MS.22R4.B1 sextupoles and a trim of the MQML.10R4.B1 individually powered quadrupole, as shown in Fig. 17. The effect of the trim on the local model is shown in Fig. 18 in the orange line. Predictions from simulations showed that this fit corrected the error without large orbits or quadrupolar trims. In

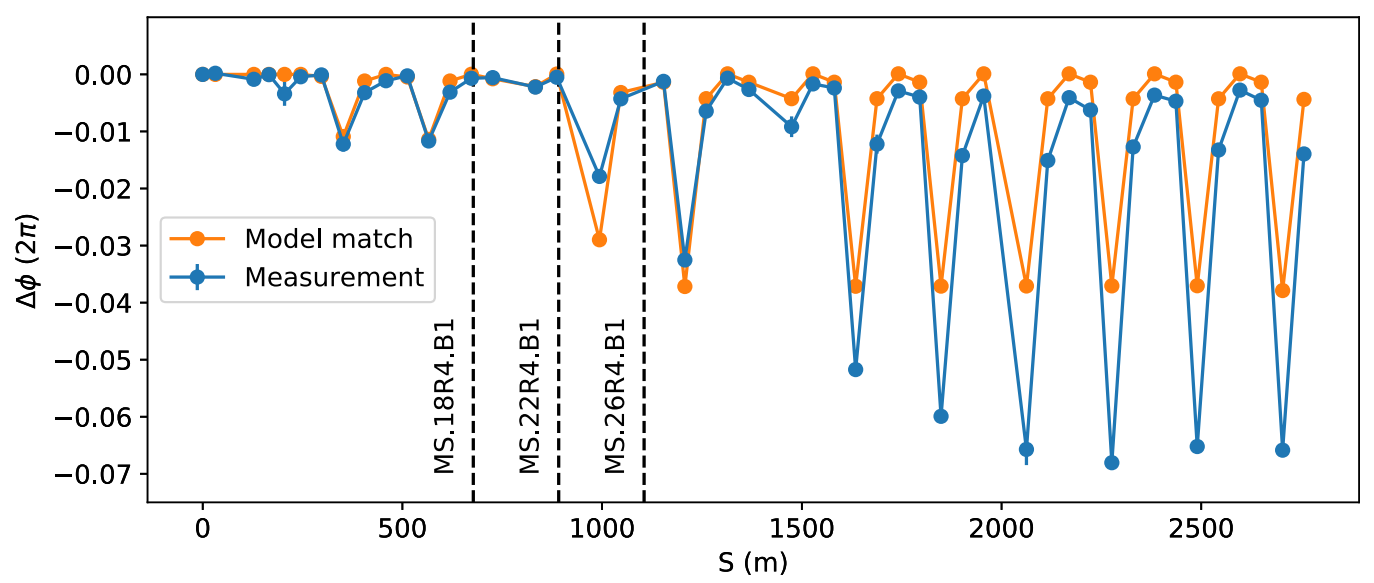

FIG. 18. Horizontal betatron phase-advance deviation from the local model in arc 45. In blue the measurement and in orange the effect of the local correction with orbit bumps in sextupoles. The sextupoles where the bump reached its peak are marked. 


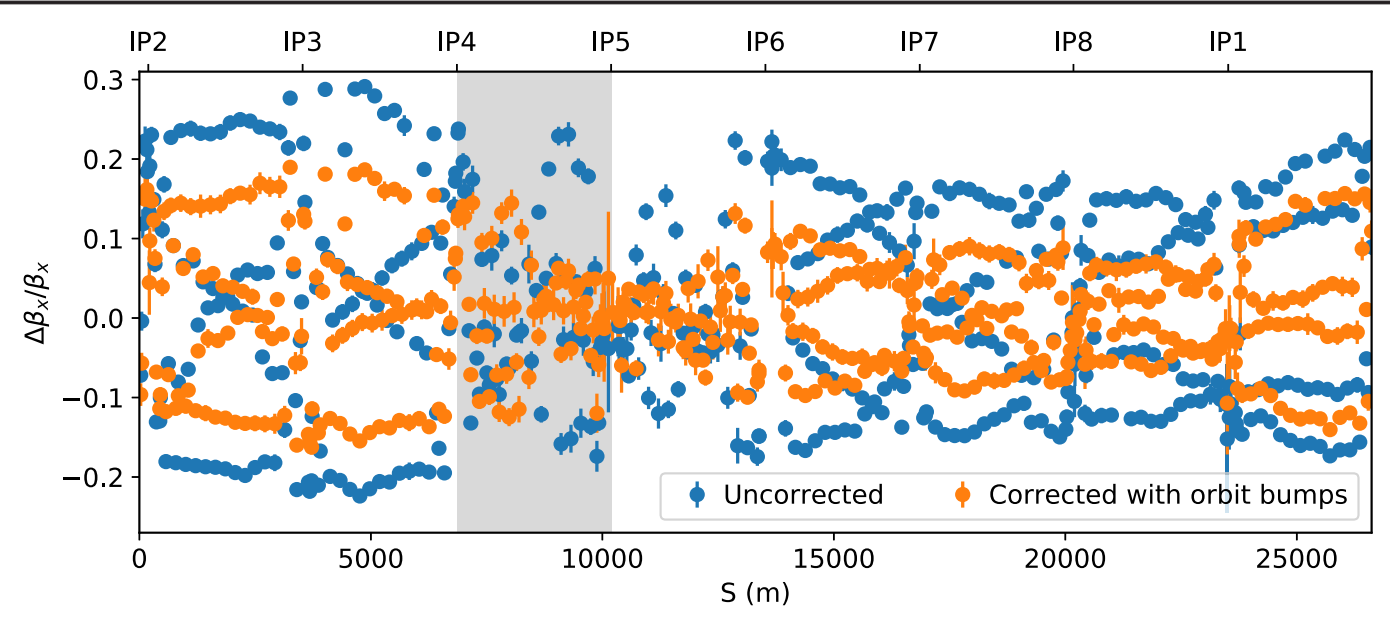

FIG. 19. Measurement of the effect on the horizontal $\beta$-beating of Beam 1 of the correction with orbit bumps in sextupoles performed during the flat optics MD that took place in 2018. The previous global corrections calculated during 2017 were removed. The grey area is the region of the accelerator targeted by the correction. The $24 \%$ peak in arc 45 was reduced to about $5 \%$ and the overall $\beta$-beating around the machine was reduced about $10 \%$.

Table III the orbit correction and trim of MQML.10R4.B1 used to produce the knob are shown.

The effect of this correction on the global $\beta$-beating can be seen in Fig. 19. The large $\beta$-beating peak in arc 45 was reduced to the $5 \%$ level and the overall $\beta$-beating in the machine was also reduced by about $10 \%$.

\section{SUMMARY}

Local corrections in the LHC interaction regions have traditionally been a manual tedious task. A new automatic local correction algorithm and tool has been developed to boost the correction quality and speed.

This algorithm has been used to perform Monte-Carlo simulations with the expected field and alignment errors of HL-LHC. It has been proven that the algorithm is able to correct down to the $\beta^{*}$ measurement uncertainty level for both beams. This shows that the factor constraining the performance of the HL-LHC from the optics point of view is the accuracy of the measurement of the $\beta^{*}$. The automatic matching routine has also been used to establish a tolerance of $\pm 1 \mathrm{mrad}$ on the maximum allowed tilts in the HL-LHC triplet quadrupoles and a waviness up to $\pm 5 \mathrm{mrad}$. This study also allowed a reduction of the integrated strength of the skew quadrupoles in IR1 and IR5, reducing the cost of the HL-LHC.

K-modulation has shown unreliable $\beta^{*}$ measurements during Run 2, which further challenges the accuracy of this technique in HL-LHC with even lower $\beta^{*}$. An improvement of this technique using the LHC tune feedback system has been explored in experiments and simulations, but ruled out due to technical limitations and the cross-talk between the Q1 beta-beating and the arc tune quadrupoles.

The phase-advance across the IP has been proven, with a phase uncertainty of $0.3 \mathrm{mrad}$ (for $\beta^{*}=50 \mathrm{~cm}$ optics), to allow a measurement of the $\beta_{w}$ using the DOROS BPM system, but blind to waist displacements. Luminosity scans with waist shifts have been tested experimentally proving that they can be used to measure the waist position with good precision. This is the first time these scans are performed successfully in a machine like the LHC, where the safety of the machine is a concern limiting the waist shift range. These two techniques combined provide accurate measurements of the $\beta_{w}$ and the waist position, resulting in the only approach to guarantee the HL-LHC performance and keep the imbalance between IP1 and IP5 within tolerable levels. HL-LHC will therefore require a totally different IR optics commissioning strategy than currently in the LHC.

It has been shown experimentally that the ATS optics scheme enhances local magnetic errors in the arcs, where local corrections are needed. The lack of individually powered correctors in those regions makes conventional local corrections with quadrupoles not viable. It has been shown experimentally that these local errors in the arcs can be corrected with orbit bumps in sextupoles via feed-down. This kind of corrections will be critical in HL-LHC that will operate with more ambitious ATS optics schemes and could cause local optics errors in the arcs to render the machine unsafe to operate.

In summary, the general HL-LHC optics correction strategy will need the new techniques experimentally demonstrated in this report which will significantly modify the traditional LHC commissioning flow as luminosity measurements will be required in intermediate stages.

\section{ACKNOWLEDGMENTS}

Research supported by the HL-LHC project. Many thanks to Stephane Fartoukh, Piotr Skowronski, Massimo Giovannozzi, Gianluigi Arduini, Lukas Malina, Elena Fol, Joschua Dilly, Ana Garcia-Tabares and all the 
members of the Optics Measurements and Corrections team for the interesting discussions and help with these studies. Also many thanks to the CERN LHC operations team and engineers in charge for the massive support given during the machine studies and commissioning source of the experimental data presented here.

[1] R. Tomás, T. Bach, R. Calaga, A. Langner, Y. I. Levinsen, E. H. Maclean, T. H. B. Persson, P. K. Skowronski, M. Strzelczyk, G. Vanbavinckhove, and R. Miyamoto, Record low $\beta$ beating in the LHC, Phys. Rev. Accel. Beams 15, 091001 (2012).

[2] T. Persson, F. Carlier, J. C. de Portugal, A. G.-T. Valdivieso, A. Langner, E. H. Maclean, L. Malina, P. Skowronski, B. Salvant, R. Tomás, and A. C. G. Bonilla, LHC optics commissioning: A journey towards $1 \%$ optics control, Phys. Rev. Accel. Beams 20, 061002 (2017).

[3] E. H. Maclean, R. Tomas Garcia, F. S. Carlier, J. M. C. De Portugal, E. Fol, K. Fuchsberger, A. G.-T. Valdivieso, M. Giovannozzi, M. Hofer, L. Malina, T. H. B. Persson, P. K. Skowronski, M. S. Camillocci, and A. Wegscheider, Detailed review of the $\mathrm{LHC}$ optics commissioning for the nonlinear era, CERN Technical Report No. CERN-ACC2019-0029 2019, https://cds.cern.ch/record/2655741.

[4] F. S. Carlier, J. C. De Portugal, S. Fartoukh, E. Fol, D. Gamba, A. Garcia-Tabares, M. Giovannozzi, M. Hofer, A. S. Langner, E. H. Maclean, L. Malina, L. E. M. Medrano, T. H. B. Persson, P. K. Skowronski, R. T. Garcia, F. Van Der Veken, and A. Wegscheider, Optics Measurements and Correction Challenges for the HL-LHC, CERN Technical Report No. CERN-ACC-2017-0088, 2017, https:// cds.cern.ch/record/2290899.

[5] The ATLAS Experiment at the CERN Large Hadron Collider, J. Instrum. 3, S08003 (2008); Also published by CERN Geneva in 2010, http://cds.cern.ch/record/ 1129811.

[6] The CMS experiment at the CERN LHC. The Compact Muon Solenoid experiment, J. Instrum. 3, S08004 (2008); Also published by CERN Geneva in 2010, http://cds.cern .ch/record/1129810.

[7] The ALICE experiment at the CERN LHC. A Large Ion Collider Experiment, J. Instrum. 3, S08002 (2008); Also published by CERN Geneva in 2010, https://cds.cern.ch/ record/1129812.

[8] F. Carlier and R. Tomás, Accuracy and feasibility of the $\beta^{*}$ measurement for lhc and high luminosity lhc using $k$ modulation, Phys. Rev. Accel. Beams 20, 011005 (2017).

[9] M. Hofer, R. Tomas, and F. Carlier, K-modulation in future high energy colliders, in Proc. 10th Int. Particle Accelerator Conf. (IPAC'19), Melbourne, Australia (2019), pp. 476479.

[10] S. Fartoukh, Achromatic telescopic squeezing scheme and application to the LHC and its luminosity upgrade, Phys. Rev. Accel. Beams 16, 111002 (2013).

[11] M. Aiba, S. Fartoukh, A. Franchi, M. Giovannozzi, V. Kain, M. Lamont, R. Tomás, G. Vanbavinckhove, J. Wenninger, F. Zimmermann, R. Calaga, and A. Morita, First $\beta$-beating measurement and optics analysis for the
CERN large hadron collider, Phys. Rev. Accel. Beams 12, 081002 (2009).

[12] R. Tomás, O. Brüning, M. Giovannozzi, P. Hagen, M. Lamont, F. Schmidt, G. Vanbavinckhove, M. Aiba, R. Calaga, and R. Miyamoto, CERN large hadron collider optics model, measurements, and corrections, Phys. Rev. Accel. Beams 13, 121004 (2010).

[13] H. Grote and F. Schmidt, MAD-X: An upgrade from MAD, in Proceedings of the 20th Particle Accelerator Conference, PAC-2003, Portland, OR, 2003 (IEEE, New York, 2003), https://mad.web.cern.ch/mad/.

[14] G. Apollinari, I. Bejar, O. Bruning, P. Fessia, M. Lamont, L. Rossi, and L. Tavian, High-Luminosity Large Hadron Collider (HL-LHC): Technical Design Report V. 0.1, CERN Yellow Reports: Monographs (CERN, Geneva, 2017), http://cds.cern.ch/record/2284929.

[15] L. E. M. Medrano, R. Tomas, G. Arduini, and M. N. Mendívil, Assessment of the performance of High Luminosity LHC operational scenarios: integrated luminosity and effective pile-up density, Can. J. Phys. 97, 498 (2019).

[16] T. H. B. Persson et al., Run 2 Optics and Corrections, 9th LHC Operations Evian Workshop, Evian, France (2019), https://indico.cern.ch/event/751857/.

[17] R. Calaga, R. Tomás, and A. Franchi, Betatron coupling: Merging hamiltonian and matrix approaches, Phys. Rev. Accel. Beams 8, 034001 (2005).

[18] T. Persson and R. Tomás, Improved control of the betatron coupling in the large hadron collider, Phys. Rev. Accel. Beams 17, 051004 (2014).

[19] H. M. Durand et al., HL-LHC alignment requirements and associated solutions, in Proc. 8th Int. Particle Accelerator Conf. (IPAC'17) (2017), pp. 1893-1896, http://dx.doi.org/ 10.18429/JACoW-IPAC2017-TUPIK085.

[20] J. M. C. De Portugal, S. Fartoukh, E. Fol, A. G.-T. Valdivieso, E. H. Maclean, L. Malina, T. H. B. Persson, P. K. Skowronski, M. S. Camillocci, R. T. Garcia, and A. Wegscheider, MD2148: Flat optics, CERN technical report, 2018, https://cds.cern.ch/record/2632141.

[21] A. Boccardi, A. Butterworth, E. C. Giraldo, R. Denz, M. Gasior, J. Gonzalez, S. Jackson, L. Jensen, O. Jones, Q. King, G. Kruk, M. Lamont, S. Page, R. Steinhagen, and J. Wenninger, Commissioning and initial performance of the LHC beam-based feedback systems, in Proceedings of the International Particle Accelerator Conference, Kyoto, Japan (ICR, Kyoto, 2010), pp. 2779-2781.

[22] M. Gasior, G. Baud, J. Olexa, and G. Valentino, First operational experience with the LHC diode orbit and oscillation (DOROS) system, in Proc. 5th Int. Beam Instrumentation Conf. (IBIC'16) (2016), pp. 43-46, http://dx.doi.org/10.18429/JACoW-IBIC2016-MOPG07.

[23] T. Persson, J. C. de Portugal, A. Garcia-Tabares, M. Gasior, A. Langner, T. Lefevre, E. Maclean, L. Malina, J. Olexa, P. Skowronski, and R. Tomas, Experience with DOROS BPMs for coupling measurement and correction, in Proc. 7th Int. Particle Accelerator Conf. (IPAC'16) (2016), pp. 303-305, http://dx.doi.org/10.18429/JACoWIPAC2016-MOPMR029.

[24] A. Morita, Status of SuperKEKB phase-2 commissioning, in Proceedings, 39th International Conference on High Energy Physics (ICHEP2018): Seoul, Korea, July 4-11, 
2018 (2019), p. 228, http://dx.doi.org/10.22323/1.340 .0228 .

[25] A. Morita, Status of SuperKEKB phase-2 commissioning, presented in the 39th International Conference on High Energy Physics (ICHEP2018), 2018, https://indico .cern.ch/event/686555/contributions/2962552/attachments/ 1681111/2700857/ichep2018_KEK_A.Morita.pdf.

[26] W. Herr and B. Muratori, Concept of luminosity, in CAS CERN Accelerator School: Intermediate Course on Accelerator Physics, Zeuthen, Germany (2003), pp. 361-378, https://cds.cern.ch/record/941318.

[27] L. van Riesen-Haupt, J. M. Coello de Portugal, E. Fol, A. Seryi, and R. Toms, K-modulation developments via simultaneous beam based alignment in the LHC, in Proc. 8th Int. Particle Accelerator Conf. (IPAC'17)
(2017), pp. 2159-2162, http://dx.doi.org/10.18429/ JACoW-IPAC2017-TUPVA042.

[28] S. Fartoukh, R. Bruce, F. Carlier, J. C. De Portugal, A. Garcia-Tabares, E. Maclean, L. Malina, A. Mereghetti, D. Mirarchi, T. Persson, M. Pojer, L. Ponce, S. Redaelli, B. Salvachua, P. Skowronski, M. Solfaroli, R. Tomás, D. Valuch, A. Wegscheider, and J. Wenninger, Experimental validation of the Achromatic Telescopic Squeezing (ATS) scheme at the LHC, J. Phys. Conf. Ser. 874, 012010 (2017).

[29] S. Fartoukh et al., LHC Beam experiments with telescopic flat optics in 2018, CERN Technical Report No. CERN-ACC-2019-0052, 2019, https://cds.cern.ch/record/ 2687343. 